\title{
Algorithm to demodulate an electromyogram signal modulated by essential tremor
}

\author{
Yuya Matsumoto ${ }^{1}$, Masatoshi Seki ${ }^{2,4}$, Yasutaka Nakashima ${ }^{2,3}$, Takeshi Ando ${ }^{2,5}$, Yo Kobayashi ${ }^{2,6^{*}}$, Hiroshi lijima ${ }^{7}$, \\ Masanori Nagaoka ${ }^{8}$ and Masakatsu G. Fujie ${ }^{2}$
}

\begin{abstract}
Essential tremor is a disorder that causes involuntary oscillations in patients both while they are engaged in actions and when maintaining a posture. Such patients face serious difficulties in performing daily living activities such as meal movement. We have been developing an electromyogram (EMG)-controlled exoskeleton to suppress tremors to support the movements of these patients. The problem is that the EMG signal of the patients is modulated by the tremor signal as multiplicative noise. In this paper, we proposed a novel signal processing method to demodulate patients'EMG signals. We modelled the multiplicative tremor signal with a powered sine wave and the tremor signal in the EMG signal was removed by dividing the modelled tremor signal into the EMG signal. To evaluate the effectiveness of the demodulation, we applied the method to a real patient's EMG signal, extracted from biceps brachii while performing an elbow flexion. We quantified the effect of the demodulation by root mean square error between two kinds of muscle torques, an estimated torque from the EMG signal and calculated torque from inverse dynamics based on the motion data. The proposed method succeeded in reducing the error by approximately $15-45 \%$ compared with using a low-pass filter, typical processing for additive noise, and showed its effectiveness in the demodulation of the patients'EMG signal.
\end{abstract}

Keywords: Assistive device, Biosignal processing, Electromyogram (EMG) signal, Essential tremor, Wearable robotics

\section{Background \\ Essential tremor}

Essential tremor is the most common pathological tremor, in which the tremor symptoms occur while the patient is performing an action or maintaining a posture. Researchers have indicated that older people often experience essential tremor. Some have reported that about $4 \%$ of the population above the age of 40 experience essential tremor [1], while others have reported it in $5-14 \%$ of individuals aged over 65 [2, 3]. Essential tremor can result in functional disability and causes social inconvenience. Approximately $65 \%$ of essential tremor patients have serious difficulties in performing daily activities such as meal movement $[4,5]$.

*Correspondence: you-k@fuji.waseda.jp

2 Faculty of Science and Engineering, Waseda University, Tokyo, Japan

Full list of author information is available at the end of the article
Current approaches for the suppression of essential tremor in practical use can be divided into two types: the use of medication to suppress the overreaction of the nerves, and the use of electrical stimulation to a specific part of the brain, which is called deep brain stimulation. However, both of these approaches have significant limitations, namely, the side effects of the medication, and the invasive nature of the implantation of electrodes into the brain, respectively. As a result, studies on alternative approaches are ongoing. Some researchers have used functional electrical stimulation to suppress the tremors [6-8]. Others have proposed various methods to suppress tremor mechanically. Yano et al. proposed an endeffector meal-assistant robot with an adaptive filter for tremor suppression $[9,10]$. Komatsuzaki et al. proposed a tremor suppression method using a shock absorber [11]. 


\section{Motivation}

As an alternative mechanical approach, we have been developing an exoskeleton for the upper limbs to support voluntary movement in essential tremor patients (see Fig. 1a) [12-17]. The goal for the exoskeleton is to support patients while they have a meal, because meals are one of the most important daily activities that are affected by tremor. The advantages of using the exoskeleton are its ease of use-patients simply need to wear itand less invasiveness compared with existing approaches. The exoskeleton consists of two frames, a frame for the forearm and a frame for the upper arm; and a body, which contains a motor, gears, and a potentiometer as shown in Fig. 1b, c. Table 1 shows the specification of the exoskeleton. The shapes of the frames are very characteristically spiral, and this spiral is designed to make the process of wearing the exoskeleton easier compared with an exoskeleton that is fixed to the wearer's body via cuffs. The spiral frames are made of aluminium alloy A5052 and are easy to manually loosen or tighten the spiral according to the user's arm. The spiral frames also have enough stiffness to constrain the wearer's elbow joint movement. The combination of motor and gears mounted on the body are selected based on the measurement of a torque produced by the oscillated upper limb and the measurement of the motion speed during drinking movement. The specification and required specification of the selected combination is also showed in Table 2. The axis of the motor is placed parallel to the rotation axis of the frame of the forearm, and the motor torque is transmitted to the frame via several gears as shown in Fig. 1c. The rotation axis of the frame of the forearm is also connected to the potentiometer with other several gears to monitor the rotation angle.

The core of the proposed exoskeleton is the control of the motor. The motor is controlled by an electromyogram
Table 1 Weight of the proposed exoskeleton

\begin{tabular}{ll}
\hline Part & Weight (g) \\
\hline Body & 210 \\
Frame & 150 \\
\hline
\end{tabular}

Table 2 Specification and required specification of geared motor

\begin{tabular}{lcl}
\hline Parameter & Specification & Required specification \\
\hline No load speed (deg/s) & 83.9 & More than 80.0 \\
Stall torque $(\mathrm{Nm})$ & 1.3 & More than 1.0 \\
\hline
\end{tabular}

(EMG) signal from the patients. EMG signals are often used as input signals to control exoskeletons because an amplitude of EMG signals are almost relative to the level of muscle activation [18-24]. Based on the estimation of the voluntary muscle activation from the amplitude of EMG signal, the motor drives along with the estimated voluntary motion; that is, when the user intends to perform an action, the motor follows the intended action, and when the user intends to maintain a posture, the motor retains its posture and constrains the joint motion. The torque of the motor is transmitted to the biological arm via one of the frames of the exoskeleton. As a first evaluation, we have already reported that the amplitude of the oscillation was reduced by about $50-80 \%$ using the proposed exoskeleton controlled with a toggle switch [17]. To reach the aforementioned final goal, as a next step, to control this exoskeleton along with the user's intention, the accurate estimation of the voluntary movement from the EMG signal of the patients is the most important remaining technical challenge.

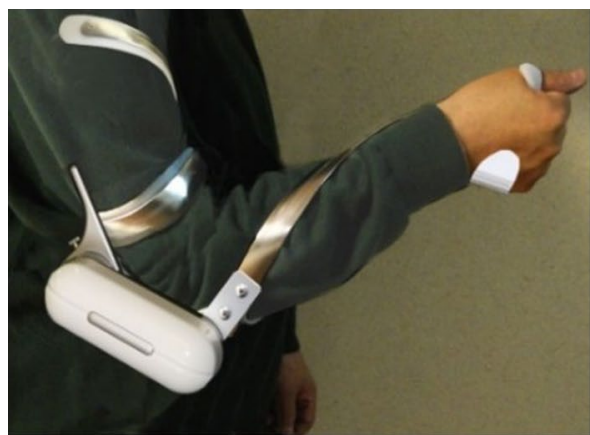

a Appearance

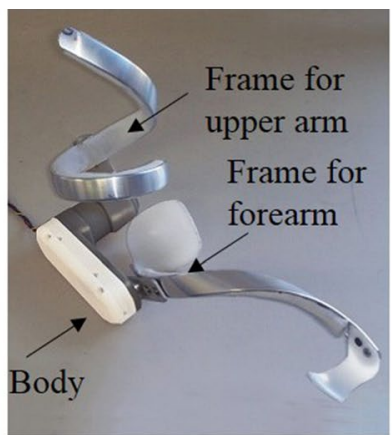

b Components

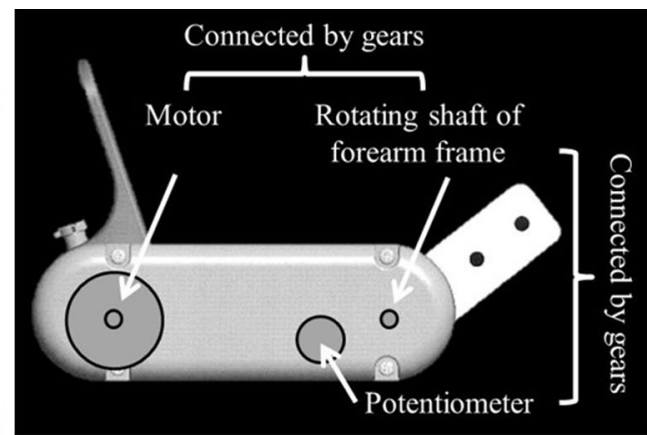

c Inner structure

Fig. 1 Exoskeleton for essential tremor patients. In $\mathbf{a}$, the appearance of the proposed exoskeleton that is worn by a user is shown, in $\mathbf{b}$, components of the exoskeleton are shown, and in $\mathbf{c}$, a picture of the inner structure of the body of the exoskeleton is shown 


\section{EMG signals of essential tremor patients}

The point of estimating the voluntary movements from the patients' EMG signal is to remove the signal causing the involuntary oscillation in the EMG signal. It is well known that EMG signals of patients with tremors contain not only voluntary commands but also involuntary tremor signals. Figure 2 shows the raw EMG signal from an essential tremor patient. In the area emphasized with the black dashed circle, there are intermittent EMG bursts. This intermittent series of bursts is called a "grouped discharge", and is a typical signal from tremor patients. The intermittent bursts alternately cause muscle contraction and relaxation and result in the oscillation of the body. The frequency of the intermittent bursts is patient dependent, but is within a certain range; some authors report 4-12 Hz [25], while others report 5-8 Hz (Fig. 2) [26]. Additionally, we have observed that the characteristic of the shape of these intermittent bursts varies depending on the patient's movement state, i.e., whether performing an action or maintaining a posture [16]. From the categorization of noise, there are two types of noise: additive noise and multiplicative noise. Figure 3 shows examples of the signal shape of each noise type. In the case of additive noise, noise is added to the input signal, and in the case of multiplicative noise, noise is multiplied by the input signal. It has been argued that tremor signals modulate the EMG of the tremor patients as multiplicative noise $[27,28]$.

Some approaches to tremor signal removal have been proposed by previous researchers. Rocon et al. proposed an adaptive filter with a weighted-frequency Fourier linear combiner for the force and angular velocity data that they used for their proposed exoskeleton for tremor patients [29]. Yano et al. developed an adaptive filter for force sensor data that was used in admittance control for a meal-assistance manipulator [9, 10]. This filter estimated the tremor frequency and attenuated the signal in the estimated frequency band using a band stop filter. Riviere et al. proposed a filtering algorithm for physiological tremors that arise during microsurgery [30]. However, these studies aimed to reduce tremor noise in motion signals that were measured by sensors such as force sensors or position meters. In these signals, the effects of the tremors are observed as additive noise. Therefore, it is difficult to apply these methods to the patients' EMG signal.

To remove multiplicative noise, the cepstral mean normalization [31] (CMN) and maximum a posteriori estimation CMN (MAP-CMN) methods are widely used in the field of speech recognition. However, CMN is not a real-time adaptive method, and MAP-CMN requires cepstral mean calculations based on a signal of sufficient length to provide superior performance. It is difficult to use such methods for controlling the exoskeleton, because the acceptable estimation delay for controlling the exoskeleton is shorter than that of speech recognition; the exoskeleton must follow the user's motion as fluently as possible. Additionally, to design the removal filter for the multiplicative tremor signal during a motion, it is essential to adapt the filter for the characteristics of the tremor signal during the motion. However, there are almost no studies that focus on the characteristics of the multiplicative tremor noise during a particular motion except for our previous study $[15,16]$, because the main objectives of the related studies on the characteristics of the tremor signal in EMG were to use the signal information to diagnose the cause of tremor. Therefore, to control the exoskeleton accurately based on the EMGs of essential tremor patients during a meal movement, it is necessary to develop an approach that can treat a signal



Fig. 2 Features of the EMG signals of essential tremor patients. These signals were measured from biceps brachii of real essential tremor patients when they performed elbow flexion. The red line shows the EMG signal and the black line shows the elbow angle 



Fig. 3 Examples of two types of noise $\left(f_{1}=5[\mathrm{~Hz}], f_{2}=50[\mathrm{~Hz}]\right)$. a Additive noise $\left(\sin 2 \pi f_{1} t+\sin 2 \pi f_{2} t\right)$. b Multiplicative noise $\left(\sin 2 \pi f_{1} t * \sin 2 \pi f_{2} t\right)$

with multiplicative noise in real time and adapt it to the characteristics of the tremor signal during the movement.

\section{Objective}

The objective of this paper was to propose a real-time processing method to demodulate the EMG signal of essential tremor patients, and to evaluate its effectiveness with a real patient's EMG signal. To demodulate the EMG signal, we modelled the multiplicative tremor noise with a powered sine wave and divided the modelled multiplicative noise into the EMG signal. Based on the characteristics of the tremor signal, to evaluate the effectiveness of the proposed method, the following two points must be discussed:

- Whether the proposed method successfully demodulates the real patient's EMG signal.

- Whether the proposed method works well for both signals extracted while performing an action and while maintaining a posture, because essential tremor is a postural and an action tremor, and the characteristics of the signal vary based on the movement state.

\section{Methods}

In this section, we describe the detail about the proposed demodulation algorithm and describe how to evaluate the effect of the proposed method.

\section{Concept of the proposed method}

In the first half of "Methods", we describe the proposed real-time filtering algorithm used to demodulate the EMG signals of essential tremor patients. As we described in "Background", the tremor signal in the EMG signals is multiplicative noise, and it is difficult to process multiplicative noise with methods for additive noise. Figure 4 shows a simple example demonstrating that typical low-pass filters (LPF) and high-pass filters (HPF) do not work for multiplicative noise. In Fig. 4, a secondorder Butterworth filter with a cutoff frequency of $20 \mathrm{~Hz}$ was used as an LPF and HPF, and was used to process both additive and multiplicative noise. From Fig. 4, it is clear that the typical LPF and HPF extract the ideal signal when additive noise is inputted, but that they do not work for multiplicative noise. To process the EMG signal of the patients in real time, we focused on the fact that the tremor signal is multiplied by the voluntary signal. In other words, if the strength of the modulation induced by the tremor signal can be estimated, then the effects of the tremor signal can be removed by dividing the estimated modulation strength into the EMG signal of the patients as follows:

$$
s_{n}=\frac{1}{N_{D}} \cdot e_{n},
$$

where $s_{n}$ is the filtered signal, $n$ is the number of samples, $N_{D}$ is the estimated modulation strength by the tremor signal, and $e_{n}$ is the measured EMG signal of the patient.

\section{Model of the tremor signal}

First, to estimate the modulation strength in real time, it is important that the modulation by the tremor signal is expressed with a mathematical model. The mathematical model needs to fulfil the following conditions:

- The model needs to represent the periodic characteristic of the tremor signals, in which the amplitude rises and falls alternately.

- The modulation strength $N_{D}$ must be greater than zero because when the modulation strength $N_{D}$ is zero, the filtered signal $s_{n}$ goes to infinity in Eq. (1). 

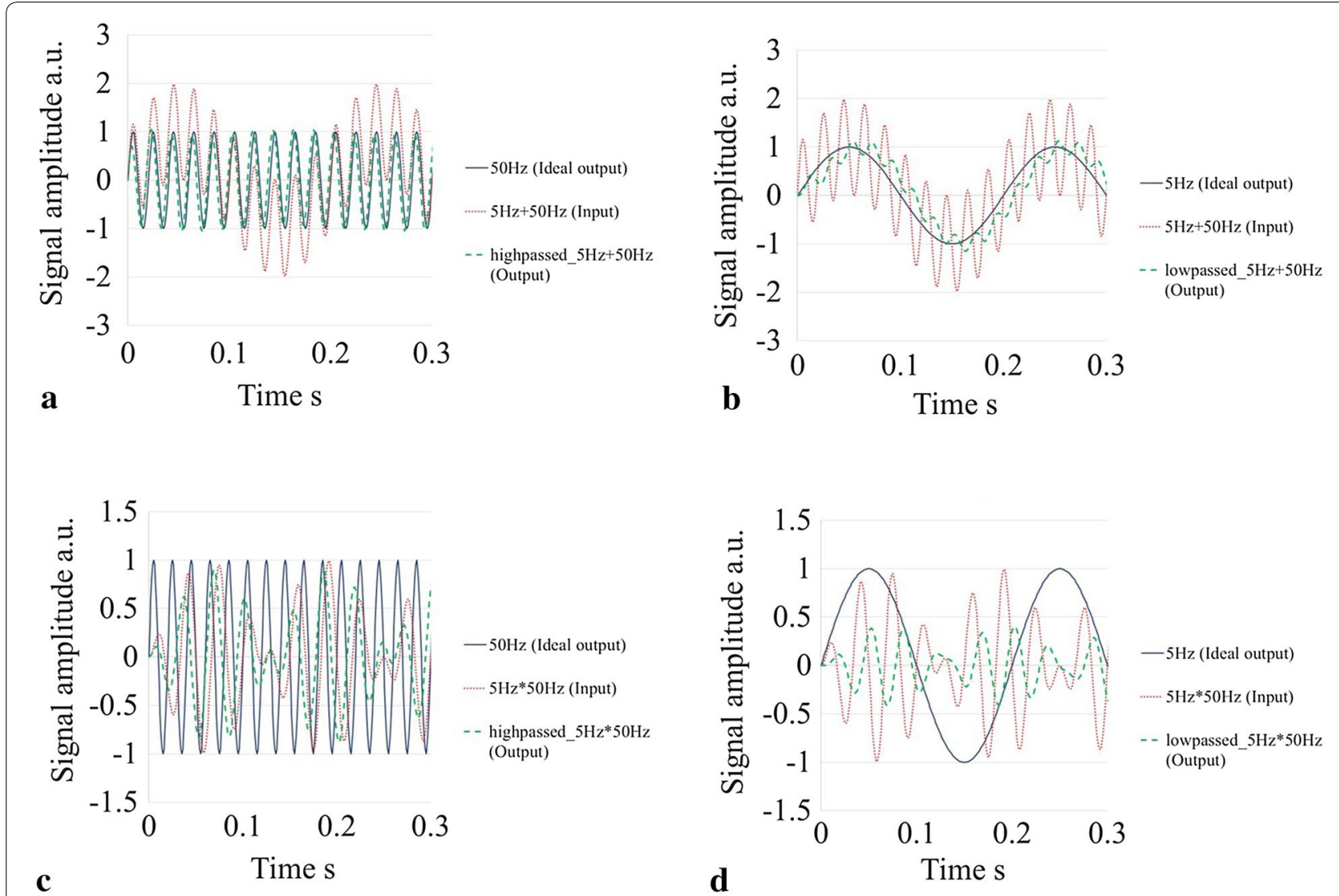

Fig. 4 Effect of high-pass filter (HPF) and low-pass filter (LPF) for two types of noise. In this example, a second-order Butterworth filter with a cutoff frequency of $20 \mathrm{~Hz}$ is used as both an HPF and an LPF to process two kinds of noise. In all four graphs, the red dotted line shows the input signal, which is made of two frequency component signals, one being additive and the other multiplicative; the green dashed line shows the output signal of the filters; and the dark blue line shows the ideal output of the filters (in the case of using an LPF, a low-frequency component is ideal output, and in the case of using an HPF, a high-frequency component is ideal output). a Additive noise processed with HPF. b Additive noise processed with LPF. c Multiplicative noise processed with HPF. d Multiplicative noise processed with LPF

- When the EMG signal of the essential tremor patients are not modulated, the modulation strength $N_{D}$ needs to equal one because the filtered signal equals the measured EMG signal.

There are several studies that have constructed such a mathematical model $[27,28]$. In this study, we constructed the model based on a model proposed by Bacher et al. [28] because the effectiveness of their proposed method was evaluated by a comparison between a real patient's EMG and a simulated signal that was made by multiplying Gaussian white noise by their proposed tremor model. In this study, we estimated the modulation strength $N_{D}$ of the tremor signal as follows:

$N_{D}=1-f\left(C_{M A X}\right) \cdot M+2 \cdot f\left(C_{M A X}\right) \cdot M \cdot \sin ^{k} \theta_{T}$,

where $f\left(C_{\text {MAX }}\right)$ is a sigmoid function, $M$ is the modulation depth $(0 \leq M<1), k$ is the order of the powered sine wave, and $\theta_{T}$ is the phase of the powered sine wave. The third term is a powered sine wave, which simulates the periodic characteristics of the tremor signal. The modulation depth $M(0 \leq \mathrm{M}<1)$ and the sigmoid function $f\left(C_{M A X}\right)$ work as gains of the simulated tremor signal because they are included in the gains of the powered sine wave. Figure 5 shows an example of the modulation strength $N_{D}$ with the proposed mathematical model. From Fig. 5, the modulation strength has periodic characteristics, and is always greater than zero. Furthermore, if the gain of the simulated tremor signal, the modulation depth $M$, or the sigmoid function $f\left(C_{M A X}\right)$ equals zero, then the modulation strength equals one. The proposed model fulfils all of these conditions.

Compared with previous work by Bacher et al. [28], we added a sigmoid function $f\left(C_{M A X}\right)$ as a threshold function to implement the proposed algorithm only when the EMG signal is modulated by tremor. Further, we used a powered sine wave instead of a sine wave because a 


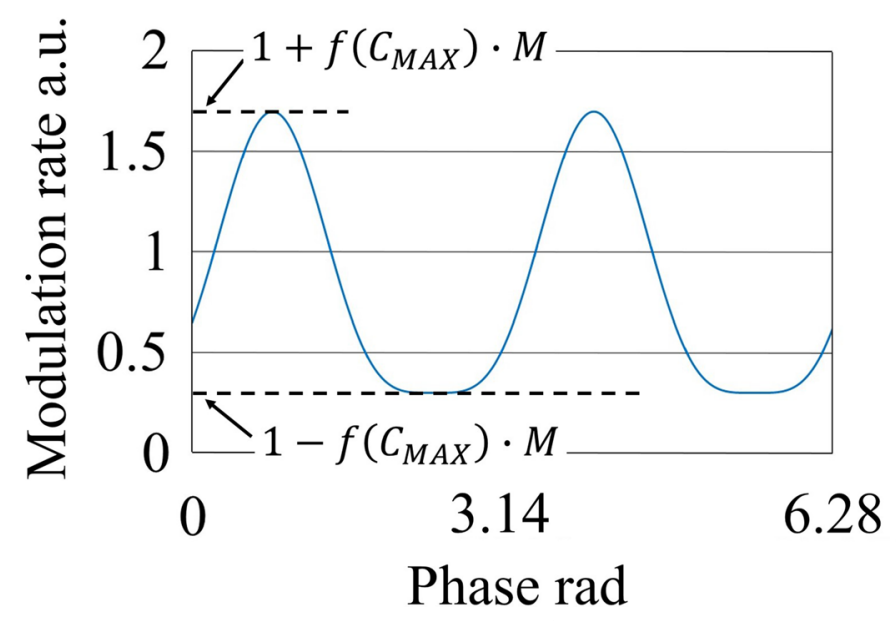

Fig. 5 Example of the variation of modulation strength $N_{D}$ against the variation of phase

powered sine wave worked well for demodulation by trial and error.

To use the model for real-time demodulation, we needed to define some of parameters in (2). How to calculate the parameters is described in the following subsections.

\section{Phase detection of powered sine wave}

The phase of the powered sine wave $\theta_{T}$ is one of the parameters that needs to be detected. To estimate the phase of the tremor signal that was present, the algorithm searched for the maximum correlation $C_{M A X}$ between the phase of the powered sine wave vector $B_{m}(m=1,2$, $\ldots, N$ ) and that of the windowed EMG vector $E_{n}$ (Fig. 3). The correlation function is expressed as follows, using Eqs. (3), (4), (5), (6), and (7).

$$
\begin{aligned}
& C=\frac{E_{n} \cdot B_{m}}{\left|E_{n}\right|\left|B_{m}\right|} \\
& E_{n}=\left\{e_{n-N}, e_{n-N+1}, \ldots, e_{n}\right\} \\
& B_{m}=\{b(m-N), b(m-N+1), \ldots, b(m)\} \\
& b(x)=\sin ^{k}(x F \Delta t \pi) \\
& \mathrm{N}=\left|\frac{1}{F \Delta t}\right|
\end{aligned}
$$

Here, $\Delta t$ is the sampling time and is set to $0.001 \mathrm{~ms}$. $B_{m}$ is one period of the powered sine wave, beginning at phase $(m-N-1) F \Delta t \pi . E_{n}$ is the present and past $1 / F \mathrm{~s}$ sampled EMG data. $F$ is the tremor frequency. Figure 6 shows how to search the present phase. Here, the windowed EMG signals were pre-processed with a HPF (cutoff: $20 \mathrm{~Hz}$ ) to remove the DC component of the signal, followed by rectification and a LPF (cutoff: $30 \mathrm{~Hz}$ ). The HPF and the LPF were Chebyshev II type filters, with damping coefficients of 0.7 .

\section{Sigmoid function}

To implement the demodulation algorithm only for signals that are affected by tremor signals, we included a sigmoid function as a threshold function in the equation for the tremor signal estimation, as shown in Eq. (2). The sigmoid function is as follows:

$$
f\left(C_{M A X}\right)=\frac{1}{1+\exp \left(-a\left(C_{M A X}-o f f s e t\right)\right)}
$$

The gain $a$ of the sigmoid function was set at 100. The offset value was set at 0.8 . Figure 7 shows the shape of the sigmoid function.

\section{Tremor frequency}

The tremor frequency $F$ is one parameter that must be defined to demodulate the EMG signal. The tremor frequency of an individual patient varies, depending on the movement state of the patient (i.e., whether the patient is performing a voluntary movement or maintaining a posture) $[15,16]$; however, the range of the variation is small. Therefore, the tremor frequency was set at $5 \mathrm{~Hz}$, which is the main tremor frequency of the patient in the experiment. This parameter needs to be set individually.

\section{Modulation depth}

As described in "Model of the tremor signal", the modulation depth is one of the gains of the powered sine wave and represents how strongly the EMG signal is modulated. When $M$ is zero, the estimated modulation strength $N_{D}$ becomes 1, according to Eq. (2) (i.e., the EMG signal is not modulated). As $M$ increases, the 

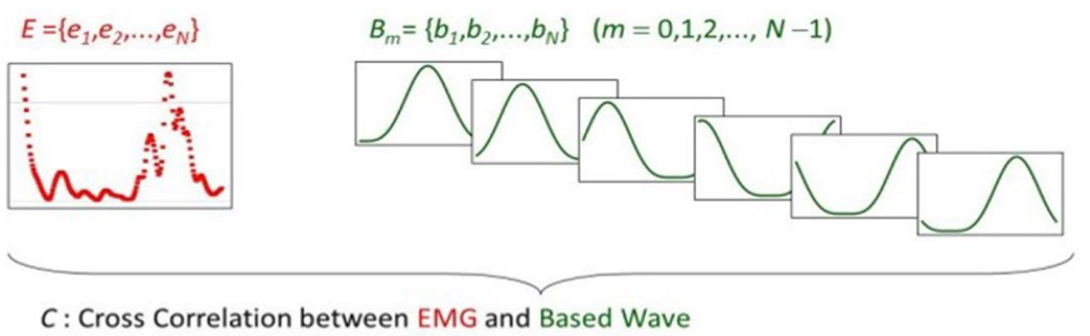

$$
C_{m}=\frac{E \cdot B_{m}}{\left|E \| B_{m}\right|}
$$

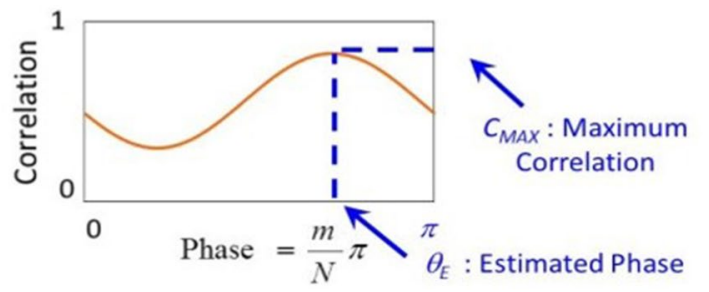

Fig. 6 Phase estimation algorithm. This algorithm searches for the maximum correlation between the base wave and the windowed EMG



Fig. 7 Sigmoid function included in the proposed algorithm. The sigmoid function prevents implementation of the proposed algorithm for signals that have not been modulated by tremor noise

amplitude of the estimated modulation strength varies, depending on the phase, as shown in Fig. 8. $M$ has a major effect on the modulation, because it is one of the gains of (2). Then, the gap between the modulation depth of a powered sine wave and that of the tremor signal in the EMG leads to severe demodulation errors. Therefore, $M$ must be fitted to an appropriate value.

We calculated $M$ by comparing the top and bottom amplitudes of the modulation strength, given by the third term of Eq. (2), with those of the EMG signal of the real ET patients as follows:

$$
\begin{aligned}
& \frac{A_{T}}{A_{B}}=\frac{1+f\left(C_{M A X}\right) \cdot M}{1-f\left(C_{M A X}\right) \cdot M} \\
& \therefore M=\frac{A_{T}-A_{B}}{f\left(C_{M A X}\right) \cdot\left(A_{T}+A_{B}\right)},
\end{aligned}
$$

where $A_{T}$ and $A_{B}$ refer to the top and bottom amplitudes of the tremor signal in the EMG, respectively. Furthermore, the top amplitude of the modulation strength is
$1+f\left(C_{\text {MAX }}\right) * M$, and the bottom is $1-f\left(C_{\text {MAX }}\right) * M$ as shown in Fig. 5.

\section{Order of the powered sine wave}

The order of the powered sine wave $k$ is another parameter of the estimated tremor signal. We tested several values for the preliminary value of the order and found that a squared sine wave was the best wave for the demodulation process, and that the effect of changing the order was small. Therefore, we used the squared sine wave.

\section{Experimental purpose}

In this subsection, we describe the methodology of the experiment. In this experiment, we evaluated the effect of the proposed demodulation algorithm using EMG signals from an essential tremor patient. Here, although the proposed algorithm was constructed to use them in real time, in this experiment, the EMG signals were processed in off-line. To evaluate the effect of the proposed algorithm, both the signal that has been demodulated from a 

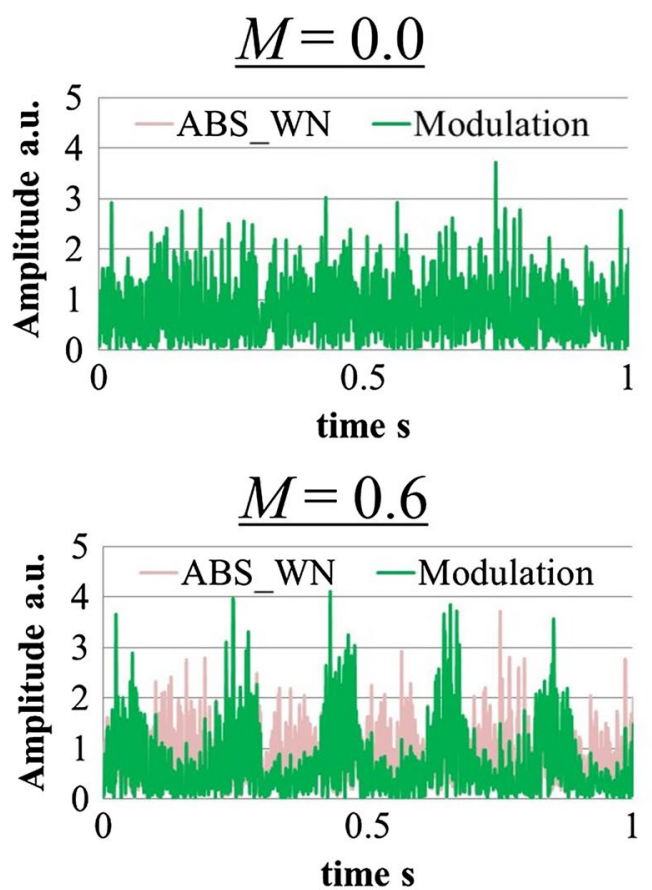
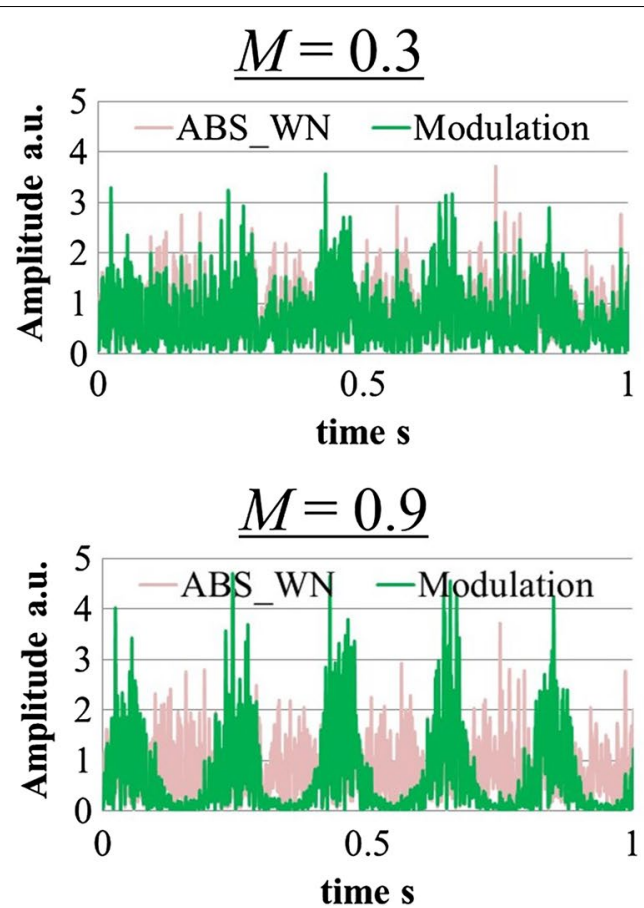

Fig. 8 Effects of the variation of the modulation strength $N_{D}$ by the variation of the modulation depth $M$. The light flesh-coloured line shows the rectified white noise, and the deep green line shows the modulated signal with the modulation strength $N_{D}$

modulated signal and the original signal prior to modulation are needed. However, the EMG signals of essential tremor patients have already been modulated when the EMG is measured, because the cause of essential tremor is thought to be a central oscillator (Fig. 9) [25]. Therefore, it is impossible to evaluate the effect of the algorithm by comparing a filtered EMG with the original EMG.

Instead, we focused on the torque produced by a muscle. The muscle torque can be assessed in two ways: by measuring the EMG of the patient, and by considering the posture and the load on the upper limb of the patient as a flowchart, as shown in Fig. 10. The first is the torque estimated from the EMG signal, and the second is the torque calculated by solving an inverse dynamics problem using information about the limb's posture and load. Therefore, in this experiment, we defined the muscle torque that was estimated from the EMG signal as the estimated data and defined the muscle torque that was calculated by inverse dynamics as the ground truth data. To evaluate the accuracy of the demodulation process, we used the root mean square error (RMSE) as a parameter; the RMSE is most commonly used to evaluate the error between two signals, and some researchers use the RMSE to evaluate the accuracy of estimation from EMG signals, e.g., [23, 32-34]. The RMSE can be calculated using the estimated data vectors $\left\{x_{1}, x_{2}, \ldots, x_{N}\right\}$ and the ground truth data vectors $\left\{\mathrm{y}_{1}, \mathrm{y}_{2}, \ldots, \mathrm{y}_{N}\right\}$ as follows:

$$
R M S E=\sqrt{\frac{1}{N} \sum_{i=1}^{N}\left(y_{i}-x_{i}\right)^{2}}
$$

We prepared three conditions of the estimated data as follows:

1. Processed with an LPF only (cutoff $=10 \mathrm{~Hz}$ ).

2. Processed with an LPF (cutoff $=10 \mathrm{~Hz}$ ) and the demodulation filter with constant parameters.

3. Processed with an LPF (cutoff $=10 \mathrm{~Hz}$ ) and the demodulation filter with the proposed parameter setting.

The estimated data (2) was the control condition of the estimated data (3), and was set to evaluate the effect on adapting modulation depth, $M$. Table 3 shows the comparison of parameters between the previous method and the proposed method.

\section{Test participant}

The participant in this experiment was an essential tremor patient (male, 70 years old) who had tremor symptoms, particularly in forearm rotation and in elbow flexion/extension. The tremor signals in the EMG signal were measured from the biceps brachii and the 


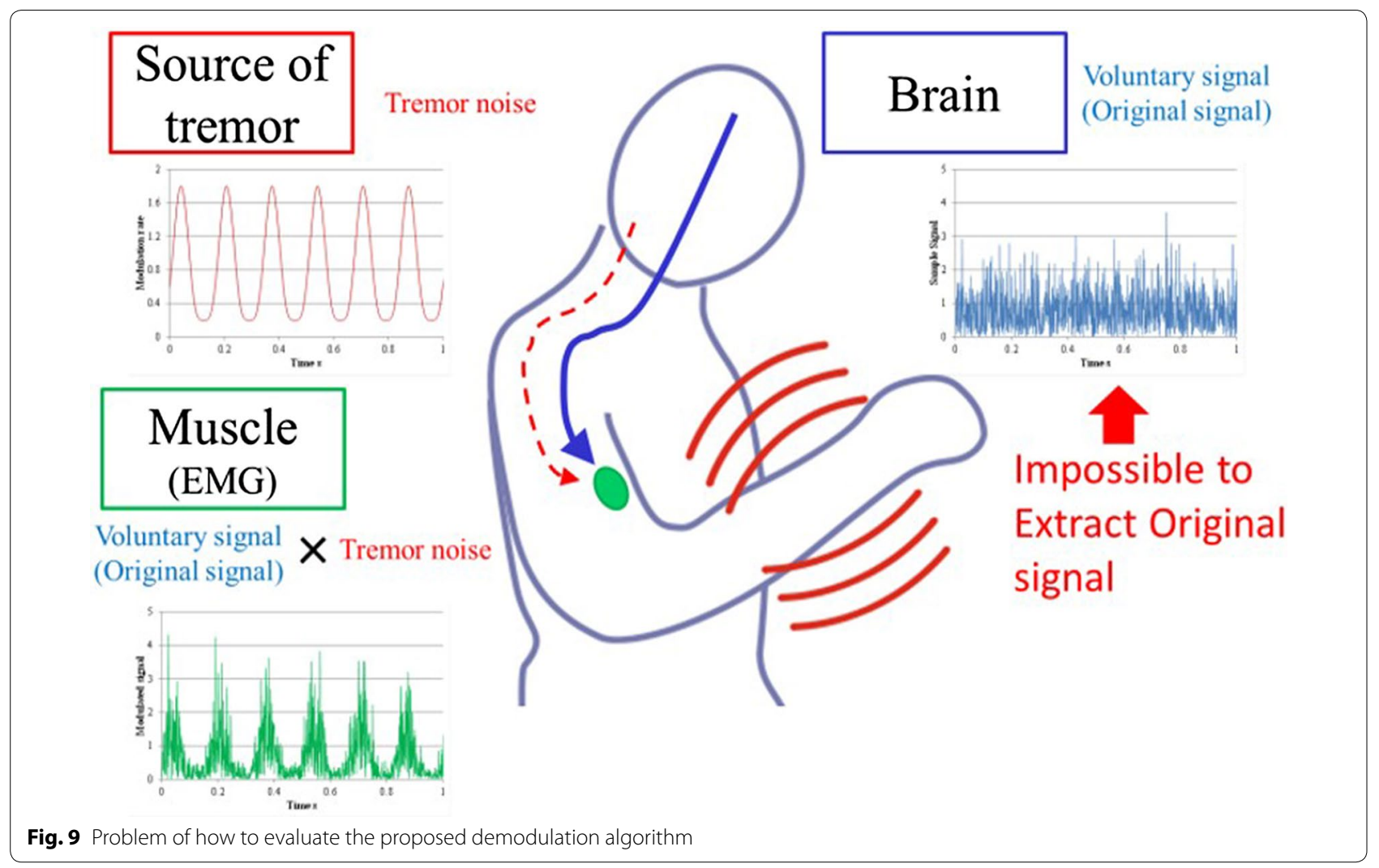

main signal frequency was approximately $5 \mathrm{~Hz}$. We gave the participant a detailed account of our experimental objectives, made it clear that he was entitled to stop the experiment whenever he desired, and obtained his consent. This experiment was approved by the Institutional Review Board of Waseda University (Approval Number: 2012-196).

\section{Experimental task}

The participant performed an elbow flexion movement while holding a bottle to simulate the movement during the process of drinking water, which is one of the target movements of the proposed exoskeleton. There were three bottles: an empty bottle, a half-full bottle, and a full bottle. The weights of these bottles are described in Table 4. The participant had practiced the elbow flexion movement in advance of the experiment to get used to the movement. At this point, the target movement time was set at $1.25 \mathrm{~s}$ throughout the practice session. The participant performed the experimental movement 10 times with each of the three bottles (for a total of 30 times). We set a short rest period (of about $3 \mathrm{~s}$ ) before the start of each motion. In this experiment, we measured two parameters: the EMG signal of the patient and the angle of the elbow joint during the task. The EMG signals were obtained using surface electrodes (Biometrics Ltd.) and a DataLog unit (Biometrics Ltd.) and were sampled at a rate of $1000 \mathrm{~Hz}$. The electrodes were placed on the biceps brachii, and the electrode positions were determined by an occupational therapist. The angle of the elbow joint was measured using the FASTRAK ${ }^{\circledR}$ magnetic position sensor (Polhemus) and was also sampled at a rate of $1000 \mathrm{~Hz}$. We pasted three markers at the acromion P1 $\left(x_{1}\right.$, $\left.y_{1}, z_{1}\right)$, the olecranon $\mathrm{P} 2\left(x_{2}, y_{2}, z_{2}\right)$, and the styloid process P3 $\left(x_{3}, y_{3}, z_{3}\right)$. The positions of the sensors are shown in Fig. 11.

\section{Muscle torque estimated from EMG signal}

In this experiment, we estimated the muscle torque from the EMG signal as follows:

$$
T_{E M G}(t, \theta)=K * E M G(t) * \alpha(\theta),
$$

where $T_{E M G}(t, \theta)$ is the estimated muscle torque, $K$ is the gain, $E M G(t)$ is the EMG signal processed with methods in each conditions [(1)-(3)] at time $t$, and $\alpha(\theta)$ is the moment of the arm at an elbow joint angle of $\theta$. According to [35], the moment $\operatorname{arm} \alpha(\theta)$ was calculated as follows:

$$
\begin{aligned}
\alpha(\theta)= & -2.988 \cdot 10^{-5} \cdot \theta^{3}+1.805 \cdot 10^{-3} \cdot \theta^{2} \\
& +4.532 \cdot 10^{-1} \cdot \theta+14.66
\end{aligned}
$$


EMG

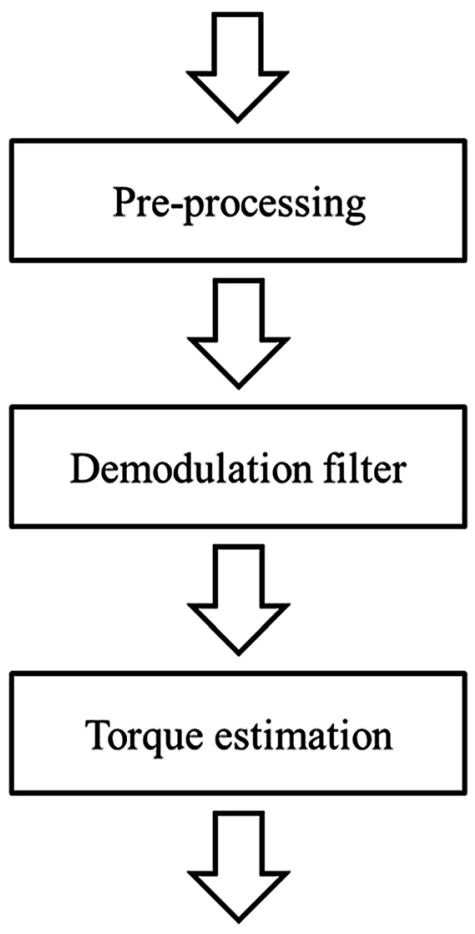

Estimated

muscle torque

\section{Position \& loading}

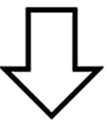

\section{Calculate joint angle}
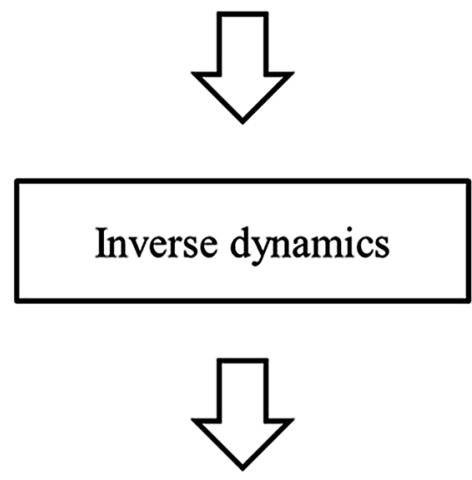

\section{Calculated}

muscle torque



\section{Calculating RMSE of}

these two muscle torque

Fig. 10 Flow to evaluate the proposed demodulation algorithm

Table 3 Parameter values of the previous and the proposed methods

\begin{tabular}{llll}
\hline Symbol & Detail & Previous & Proposal \\
\hline$f$ & Frequency $(\mathrm{Hz})$ & 5.0 & 5.0 \\
$M$ & Modulation depth (a.u.) & 0.6 & Variable \\
$k$ & Order of the powered sin wave (a.u.) & 2 & 2 \\
\hline
\end{tabular}

\section{Muscle torque calculated by inverse dynamics}

To calculate the muscle torque of the biceps brachii, we considered the equilibrium of the force around the elbow joint as follows:

$I \ddot{\theta}=T_{I}(\theta)-\left(m_{f}+m_{h}+m_{o}\right) \cdot l_{C O G} \cdot \sin (\theta+\varphi)-B \dot{\theta}$, where $I$ is the moment of inertia, $T_{I}(\theta)$ is the calculated muscle torque, and $m_{f}, m_{h}$, and $m_{o}$ are the mass of the forearm, the mass of the participant's hand, and the mass of the bottle grasped with the participant's hand, respectively. $l_{C O G}$ is the distance from the elbow joint to the centre of gravity (COG) of the forearm, hand, and bottle. $B$ is the coefficient of viscosity. Therefore, the first term of Eq. (13) is the torque of the biceps brachii, the second term is the gravity term, and the third term is the viscosity term. Here, in most cases, the triceps brachii is taken into account for the equilibrium of the force around the elbow joint. However, the triceps brachii is rarely activated in this task, and thus we excluded the torque produced by the triceps brachii. By transforming Eq. (13), the muscle torque can be calculated as follows: 
Table 4 Body weight of the patient and weight of the inertial load

\begin{tabular}{lll}
\hline Symbol & Detail & Property \\
\hline$W$ & Body weight of the patient $(\mathrm{kg})$ & 77.0 \\
$m_{0}$ & Weight of bottle $(\mathrm{kg})$ & 0.017 (empty bottle) \\
& & 0.337 (half full bottle) \\
& 0.575 (full bottle) \\
\hline
\end{tabular}

$T_{I}(\theta)=\left(m_{f}+m_{h}+m_{o}\right) \cdot l_{C O G} \cdot \sin (\theta+\varphi)+I \ddot{\theta}+B \dot{\theta}$

\section{Gravity term}

The mass of the forearm $m_{f}$ and the mass of the hand $m_{h}$ were calculated as follows:

$$
\begin{aligned}
& m_{f}=\lambda_{f} \cdot w, \\
& m_{h}=\lambda_{h} \cdot w,
\end{aligned}
$$

where $\lambda_{f}$ and $\lambda_{h}$ are the mass ratios of the forearm and the hand, respectively, which were set at constant values, according to [35], as shown in Table 5. $w$ is the body weight of the patient. Table 4 shows the body weight of the patient and the mass of the bottle.

The distance from the elbow joint to the COG of the forearm, hand, and bottle was calculated as follows:

$$
l_{C O G}=\frac{m_{f} * l_{f \_C O G}+m_{h} * l_{h_{-} C O G}+m_{o} * l_{o_{-} C O G}}{m_{f}+m_{h}+m_{o}},
$$

where $l_{f_{-} C O G}, l_{h_{-} C O G}$, and $l_{o_{-} C O G}$ refer to the distance from the elbow joint to the COG of the forearm, to the COG of the hand, and to the COG of the bottle, respectively, and were calculated as follows:

$$
\begin{aligned}
& l_{f_{-} C O G}=C O G_{f} * l_{f} \\
& l_{h_{-} C O G}=l_{f}+C O G_{h} * l_{h} \\
& l_{o_{-} C O G}=l_{f}+l_{h}
\end{aligned}
$$

Here, $l_{f}$ and $l_{h}$ are the lengths of the forearm and the hand, respectively. $C O G_{f}$ and $C O G_{h}$ are the proportions of the COG of the forearm from elbow joint and of the hand from wrist joint, respectively. $l_{f}$ and $l_{h}$ are described in Table 6, and, following [35], COG $f$ and $C O G_{h}$ are described in Table 5.

\section{Inertia term}

The moment of inertia was calculated as follows:

$$
\begin{aligned}
I= & m_{f} *\left(k_{f}^{2}+l_{f_{-} C O G}^{2}\right) *+m_{h} *\left(k_{h}^{2}+l_{h_{-} C O G}^{2}\right) \\
& +m_{o} * l_{o_{-} C O G}^{2},
\end{aligned}
$$

where $k_{f}$ and $h_{f}$ are the radius of gyration of the forearm and that of the hand, respectively, and were calculated as follows:

$$
\begin{aligned}
& k_{f}=a_{f} \cdot l_{f}, \\
& k_{h}=a_{h} \cdot l_{h},
\end{aligned}
$$

where $a_{f}$ and $a_{h}$ are the ratio of the radius of gyration of the forearm and that of the hand, respectively. Following [35], these parameters are described in Table 5.

\section{Viscous term}

According to research that estimates the muscle torque from the EMG signal and estimates the joint angle by solving dynamics problems [36], the coefficient of viscosity was set at 0.2 .

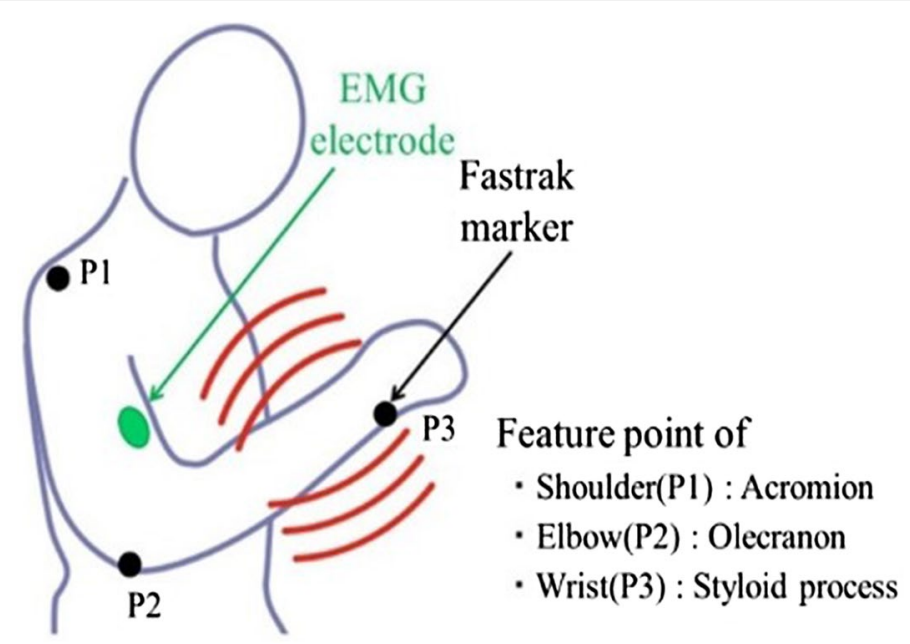

Fig. 11 Positions of EMG electrode and FASTRAK ${ }^{\circledR}$ markers 
Table 5 Physical properties of forearm and hand

\begin{tabular}{lll}
\hline Symbol & Detail & Property a.u. \\
\hline $\mathrm{COG}_{f}$ & Proportion of COG of forearm & 0.415 \\
$\lambda_{f}$ & Mass ratio of forearm & 0.016 \\
$a_{f}$ & Radius of gyration of forearm & 0.115 \\
$\mathrm{COG}_{h}$ & Proportion of COG of hand & 0.891 \\
$\lambda_{h}$ & Mass ratio of hand & 0.006 \\
$a_{h}$ & Radius of gyration of hand & 0.314 \\
\hline
\end{tabular}

Table 6 Length of parts of the patient body

\begin{tabular}{lll}
\hline Symbol & Detail & Property \\
\hline$I_{f}$ & Length of forearm $(\mathrm{m})$ & 0.250 \\
$I_{h}$ & Length of a hand $(\mathrm{m})$ & 0.125 \\
\hline
\end{tabular}

The angle of the elbow joint was calculated by using the positions of the acromion $\mathrm{P} 1\left(x_{1}, y_{1}, z_{1}\right)$, the olecranon $\mathrm{P} 2\left(x_{2}, y_{2}, z_{2}\right)$, and the styloid process $\mathrm{P} 3\left(x_{3}, y_{3}, z_{3}\right)$, as follows:

$$
\theta=\cos ^{-1}\left(\frac{x_{u} x_{f}+y_{u} y_{f}+z_{u} z_{f}}{\sqrt{x_{u}^{2}+y_{u}^{2}+z_{u}^{2}} \sqrt{x_{f}^{2}+y_{f}^{2}+z_{f}^{2}}}\right),
$$

where the values subscripted with $f$ and $u$ represent the parameters of the forearm vector and those of the upper arm vector, respectively. These two vectors were described as follows:

$$
\begin{aligned}
& \boldsymbol{r}_{\boldsymbol{f}}=\left(x_{f}, y_{f}, z_{f}\right)=\left(x_{2}-x_{1}, y_{2}-y_{1}, z_{2}-z_{1}\right) \\
& \boldsymbol{r}_{\boldsymbol{u}}=\left(x_{u}, y_{u}, z_{u}\right)=\left(x_{3}-x_{2}, y_{3}-y_{2}, z_{3}-z_{2}\right),
\end{aligned}
$$

The angle of shoulder flexion was calculated from the angle between the upper arm vector $\boldsymbol{r}_{\boldsymbol{u}}$ and the vertical line $(0,0,-1)$, as follows:

$$
\varphi=\cos ^{-1}\left(\frac{-z_{u}}{\sqrt{x_{u}^{2}+y_{u}^{2}+z_{u}^{2}}}\right),
$$

To calculate $\dot{\theta}$ and $\ddot{\theta}$, we used a pseudo derivative. The transfer function of the pseudo derivative was as follows:

$$
\frac{Y}{X}=\frac{s}{1+T_{d} \cdot s},
$$

where $T_{d}$ refers to the time constant. In this experiment, $T_{d}$ is set to $30 \mathrm{~ms}$. Here, we conducted an $s$-plane to $z$-plane transform based on the backward difference. The equation of this transform was as follows:

$$
\mathrm{s}=\frac{1-z^{-1}}{\Delta T}
$$

where $\Delta T$ refers to the sampling time, $1 \mathrm{~ms}$. Finally, Eq. (29) was substituted into Eq. (28). The calculated differential at time $t=n$ was as follows:

$$
y_{n}=\frac{x_{n}-x_{n-1}+T_{d} \cdot y_{n-1}}{\Delta T+T_{d}}
$$

where $y_{n}$ refers to the differential at $t=n$, and $x_{n}$ refers to the input signal at $t=n$.

\section{Results}

We evaluated the effects of the proposed demodulation algorithm in two movement states: first, while performing an elbow flexion, and second, while maintaining a flexed elbow posture because essential tremor is both a postural and an action tremor. Figure 12 shows one example of the processed signals. Using the estimated data, the blue line shows the muscle torque estimated from the EMG of the patient, which was processed only using an LPF (condition 1); the red dotted line shows the muscle torque that was estimated from the EMG signal that was processed by the demodulation algorithm with constant parameters and the LPF (condition 2); and the green short dashed line shows the muscle torque that was estimated from the EMG signal that was processed with the proposed demodulation algorithm and the LPF (condition 3). Using the ground truth data, the black long dashed line shows the muscle torque that was calculated from inverse dynamics. As described in the "Experimental purpose" subsection, the RMSE between the estimated data and the ground truth data was the evaluation parameter of the demodulation accuracy. Therefore, if the error between the green short dashed line and the black line is smaller than both that between the red dotted line and the black line and that between the blue line and the black line, then this means that the proposed algorithm has estimated the original signal accurately.

Figure 13 shows the results of the RMSE between the estimated data and the ground truth data. In each graph, the blue bars with diagonal patterns on the left show the results of the trial without the demodulation algorithm (condition 1), the red bars with horizontal dashed stripes in the centre show the results of the trial when processed with the demodulation algorithm with constant parameters (condition 2), and the green bars with vertical stripes on the right show the results of the trial when processed with the proposed demodulation algorithm (condition 3). The differences between the RMSEs among the three conditions were statistically evaluated against each other using the Bonferroni correction. From the graphs, while in the postural state, the RMSEs of both condition (2) and condition (3) were significantly decreased from the RMSE of condition (1), and, while in flexion, only the RMSE of condition (3) was significantly decreased from 
the RMSE of condition (1). Table 7 shows the reduction rate of the RMSE from condition (1) to condition (3). The reduction rate $R$ is calculated as follows:

$$
\mathrm{R}=\frac{R_{R M S E_{3}}}{R_{M S E_{1}}} * 100
$$

where $R M S E_{1}$ refers to the RMSE in the trial with condition (1), and $R M S E_{3}$ refers to that in the trial with condition (3). From Table 7, the reduction rate of the proposed method varied depending on the state of movement. The proposed method succeeded in reducing the RMSE by about $15-30 \%$ during a flexion movement and about $30-45 \%$ during the maintenance of a posture. Furthermore, the reduction rate varied depending on the weight of the bottle grasped during the task. The reduction rate of the proposed method was higher in the condition with the empty bottle than with the half-full bottle, and higher in this case than with the full bottle.

\section{Discussion}

\section{Effect of demodulation}

In this experiment, using the proposed method, condition (3), the RMSE between the estimated data and the ground truth data was statistically significantly decreased in all cases. Compared with the results of the control condition, condition (2), by adjusting the modulation depth $M$, the proposed method succeeded in reducing errors when the patient performed an elbow flexion in all cases. From these results, our proposed demodulation algorithm was effective for the modulated EMG signal, and the adjustment worked well for the characteristic change of the tremor signal between the postural state and the flexion.

The reason that the reduction rate varied depending on both the state of movement and the weight of the bottle is likely the influence of the voluntary signal. The voluntary signal modulates the tremor signal. As described in Section I, the tremor signal is noise in the voluntary movement signal. Conversely, the voluntary signal is also noise in the tremor signal; that is, the tremor signal is modulated strongly if patients contract their muscles strongly. This modulation made it difficult to demodulate the EMG signal, because the tremor signal could not be simulated accurately when it was modulated. Therefore, to promote the proposed demodulation algorithm, we need to analyse the characteristic change in the tremor signal depending on the voluntary signal in some way, such as a time-frequency analysis or the change in the shape of the EMG signal, and then revise the processing method.

\section{Number of participants}

In this experiment, although the number of participants was only one, the proposed method can be useful for other patients if all of the following conditions are satisfied:

- Patient-dependent parameters, such as frequency of tremor signal, are measured before the use of the proposed algorithm.

- Patients with essential tremor who have their tremor source in their biceps brachii perform actions with elbow flexion such as drinking and eating.

In the first condition, although the EMG is noisy and varies across individuals, the major characteristics of the tremor signal, "grouped discharge", is typical for the patients, and the proposed method can work for the tremor signal if patient-dependent parameters are fitted to each patient. However, in the second condition, the



Fig. 12 Comparison between the estimated data and the ground truth data 


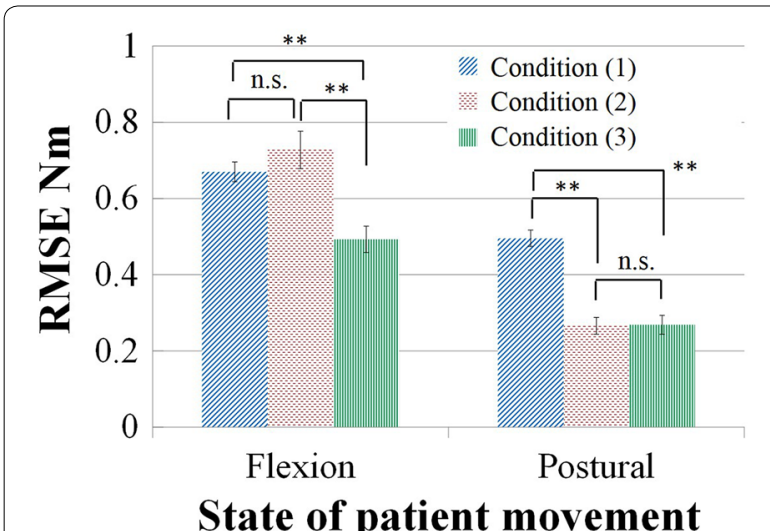

$\mathbf{a}$

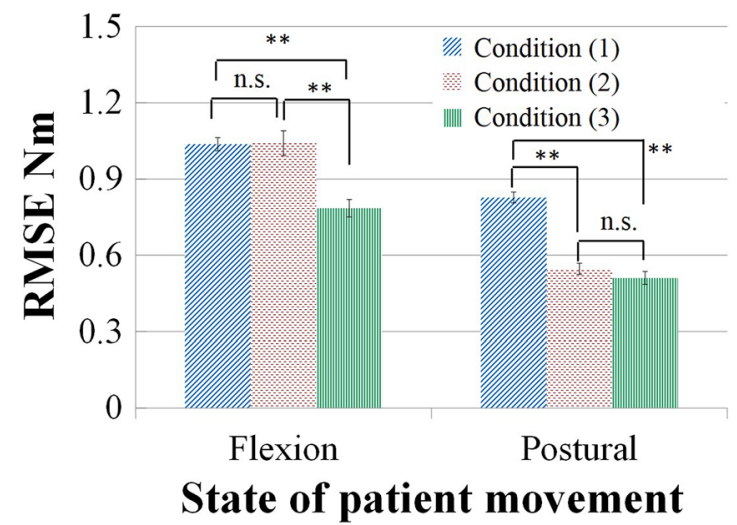

b



State of patient movement

\section{c}

Fig. 13 Results of calculation of the RMSE between the estimated data and the ground truth data. Data were statistically compared with one another using Bonferroni correction. The significance levels were adjusted based on the number of groups (the three conditions) as follows: ${ }^{*} p<0.0167(=0.05 / 3),{ }^{* *} p<0.0033(=0.01 / 3)$. a Patient holding an empty bottle. b Patient holding a half-full bottle. c Patient holding a full bottle
Table 7 Reduction rate of the RMSE

\begin{tabular}{lll}
\hline Condition of bottle & \multicolumn{2}{l}{ States of movement } \\
\cline { 2 - 3 } & Performing a flexion & Maintaining a posture \\
\hline Empty (\%) & 26.4 & 45.7 \\
Half-full (\%) & 24.3 & 38.3 \\
Full (\%) & 14.0 & 29.6 \\
\hline
\end{tabular}

evaluation did not guarantee the effectiveness of the proposed method in all the conditions because the affected muscle is patient-dependent. The proposed method only showed its effectiveness for the EMG signal from biceps brachii while performing an elbow flexion. Therefore, although the guaranteed effect from this experiment was limited, as far as the target movement of the exoskeleton, this method can be applied for demodulating the EMG signal of the patients.

\section{Effects on the control of the exoskeleton}

From these discussions, the effects of the proposed demodulation algorithm have been validated. However, we have not discussed whether the effects are sufficient for controlling the exoskeleton. To address this, we have to use the proposed method on the exoskeleton and evaluate the controlled performances of the exoskeleton while a participant is wearing it. The RMSE between the muscle torque estimated from the EMG signal and the muscle torque calculated by solving the inverse dynamics problem directs the controlled performance, because the movement of the exoskeleton is defined based on Eq. (13). We hope that the proposed method promotes the controlled performance of the exoskeleton. In future work, we will evaluate the proposed demodulation algorithm using the exoskeleton to discuss whether the effects are sufficient.

\section{Conclusions}

The objectives of this paper were to develop an algorithm to demodulate the EMG signal of essential tremor patients whose EMGs combine information about both the voluntary movement and the tremor. The EMGs of the patients is modulated by the tremor signal as multiplicative noise. Therefore, to control the exoskeleton accurately, it is essential to demodulate the EMG signal. The proposed algorithm simulates the tremor signal by approximating the tremor signal with a powered sine wave, and then divides the approximated tremor signal into the EMG signals of the patients. To simulate the tremor signal accurately, parameters of the approximation were set based on the characteristic of the real patient's EMG. Especially, we set the modulation depth, represent the gain of the modulation by tremor, as a variable to treat the characteristic change depending on the 
movement state. To evaluate the effect of the proposed method, we compared the RMSE between the muscle torque estimated from the EMG signal of the patient and the muscle torque calculated by solving the inverse dynamics problem, in three situations: without using the demodulation algorithm, using the demodulation algorithm (constant parameters), and using the proposed demodulation algorithm (modulation depth was variable). From this evaluation, we confirmed that our proposed method has a large effect on demodulating the modulated EMG signal regardless of the patient's movement state. As far as its application to the exoskeleton, the method can be applied on the EMGs of patients. However, the effect varies depending on the strength of the voluntary movement.

In future work, to improve our algorithm, we need to analyse the characteristic changes in the tremor signal depending on the strength of the voluntary signal in some way, such as time-frequency analysis or by evaluating EMG signal shape changes, and then revise the processing method. Following this, we will implement our proposed algorithm in the controller of the exoskeleton and evaluate whether the proposed algorithm is sufficient for controlling the exoskeleton.

\section{Authors' contributions \\ YM devised the basic concept of the overall system, technically developed the algorithm, carried out the experiments, analysed the data, and drafted the manuscript. MS devised the basic concept of the overall system, technically developed the algorithm, and carried out the experiments. YS, TA, and YK devised the research plan, and carried out the experiments. $\mathrm{HI}$ and $\mathrm{MN}$ drew the research design based on the clinical point of view. MF devised the basic concept and drew the research design of the overall study. All authors read and approved the final manuscript.}

\section{Author details}

${ }^{1}$ Graduate School of Creative Science and Engineering, Waseda University, Tokyo, Japan. ${ }^{2}$ Faculty of Science and Engineering, Waseda University, Tokyo, Japan. ${ }^{3}$ Faculty of Engineering, Kyushu University, Fukuoka, Japan. ${ }^{4}$ MONOZUKURI Mechatronics Laboratory, Kikuchi Seisakusho Co. Ltd., Tokyo, Japan. ${ }^{5}$ Global Manufacturing Division, Panasonic Corporation, Osaka, Japan. ${ }^{6}$ Department of Mechanical Science \& Bioengineering, Graduate School of Engineering Science, Osaka University, Osaka, Japan. ${ }^{7}$ Rehabilitation Engineering Department, Yokohama Rehabilitation Center, Yokohama, Kanagawa, Japan. ${ }^{8}$ Department of Rehabilitation Medicine, Juntendo University Graduate School, Tokyo, Japan.

\section{Acknowledgements \\ The authors sincerely thank the patient volunteer for participating in our experiment. This work was supported by the Japan Society for the Promotion of Science under a Grant-in-Aid for Scientific Research (S) (No. 25220005), a Grant-in-Aid for Challenging Exploratory Research (No. 15K12606), and a Grant-in-Aid for JSPS fellows (No. 13J05428); and by Fukushima Prefecture under the Fukushima Project on the Development of Medical and Welfare Devices, Fukushima, Japan.}

\section{Competing interests}

The authors declare that they have no competing interests.

\section{Publisher's Note}

Springer Nature remains neutral with regard to jurisdictional claims in published maps and institutional affiliations.
Received: 18 August 2016 Accepted: 13 May 2017

Published online: 31 May 2017

\section{References}

1. Dogu O, Sevim S, Camdeviren H, Sasmaz T, Buqdayci R, Aral M, Kaleaqasi H, Un S, Louis ED (2003) Prevalence of essential tremor: door-to-door neurologic exams in Mersin Province, Turkey. Neurology 61(12):1804-1806

2. Moghal S, Rajput AH, D’Arcy C, Rajput R (1994) Prevalence of movement disorders in elderly community residents. Neuroepidemiology 13:175-178

3. Louis ED, Marder K, Cote L, Wilder D, Tang MX, Lantiqua R, Gurland B, Mayeux R (1996) Prevalence of a history of shaking in persons 65 years of age and older: diagnostic and functional correlates. Mov Disord 11:63-69

4. Elble R, Koller W (1990) Tremor. Johns Hopkins Univ. Press, Baltimore

5. Rocon E, Belda-Lois J, Sanchez-Lancuesta J, Pons JL (2004) Pathological tremor management: modeling, compensatory technology and evaluation. Technol Disabil 16:13-18

6. Bó AP, Poignet P, Zhang D, Ang WT (2009) FES-controlled co-contraction strategies for pathological tremor compensation. In: The 2009 IEEE/RSJ international conference on intelligent robots and systems, October 11-15, St. Louis, USA, pp 1633-1638

7. Bo APL, Poignet P (2010) Tremor attenuation using FES-based joint stiffness control. In: IEEE international conference on robotics and automation, May 3-8, 2010, Anchorage, Alaska, USA, pp 2928-2933

8. Gallego JA, Rocon E, Pons JL (2010) Estimation of instantaneous tremor parameters for FES-based tremor suppression. In: IEEE international conference on robotics and automation, May 3-8, 2010, Anchorage, Alaska, USA, pp 1633-1638

9. Ohara E, Yano Kl, Horihata S, Aoki T, Nishimoto Y (2009) Tremor suppression control of meal-assist robot with adaptive filter. In: The 2009 IEEE international conference on rehabilitation robotics, Kyoto International Conference Center, Japan, June 23-26, 2009, pp 498-503

10. Ohara E, Yano Kl, Horihata S, Aoki T, Nishimoto Y (2009) Development of tremor-suppression filter for meal-assist robot. In: Third joint eurohaptics conference and symposium on haptic interfaces for virtual environment and teleoperator systems, Salt Lake City, UT, USA, March 18-20, 2009, pp $238-243$

11. Komatsuzaki T, Iwata Y, Ogawa K (2008) A study on vibration control of physiological tremor using dynamic absorber. Trans Jpn Soc Mech Eng Part C 74(3):679-685

12. Seki M, Matsumoto $Y$, Ando T, Kobayashi Y, lijima H, Nagaoka M, Fujie MG (2011) Development of essential tremor noise suppression filter for voluntary movement extraction from surface EMG. In: 33rd annual international conference of the IEEE EMBS, Boston, Massachusetts, USA, August 30-September 3, 2011, pp 7487-7491

13. Seki M, Matsumoto Y, Ando T, Kobayashi Y, lijima H, Nagaoka M, Fujie MG (2010) Development of robotic upper limb orthosis with tremor suppressiblity and elbow joint movability. In: 2011 IEEE international conference on systems, Man, Cybernetics, Anchorage, AK, USA, October 9-12, pp 729-735

14. Seki M, Matsumoto $Y$, Ando T, Kobayashi Y, lijima H, Nagaoka M, Fujie MG (2011) The weight load inconsistency effect on voluntary movement recognition of essential tremor patient. In: Proceedings of the 2011 IEEE international conference on robotics and biomimetics, Phuket, Thailand, December 7-11, 2011, pp 901-907

15. Matsumoto Y, Seki M, Ando T, Kobayashi Y, lijima H, Nagaoka M, Fujie MG (2012) Tremor frequency based filter to extract voluntary movement of patients with essential tremor. In: IEEE RAS \& EMBS international conference on biomedical robotics and biomechatronics, Rome, Italy, 24-27 June, 2012, pp 1415-1420

16. Matsumoto Y, Seki M, Ando T, Kobayashi Y, lijima H, Nagaoka M, Fujie MG (2012) Analysis of EMG signals of patients with essential tremor focusing on the change of tremor frequency. In: Annual international conference of IEEE Engineering in Medicine and Biology Society, SanDiego, USA, 24-27, August 28-September 2, 2012, pp 2244-2250

17. Matsumoto Y, Seki M, Ando T, Kobayashi Y, Nakashima Y, lijima H, Nagaoka M, Fujie MG (2013) Development of an exoskeleton to support 
eating movement in patients with essential tremor. J Robot Mechatron 25(6):949-958

18. Hasegawa Y, Mikami Y, Watanabe K, Firouzimehr Z, Sankai Y (2008) Wearable handling support system for paralyzed patient. In: IEEE/RSJ IEEE/RS. international conference on intelligent robots and systems, Nice, France, September 22-26, 2008, pp 741-746

19. Kiguchi K, Imada Y, Liyanage M (2007) EMG-based neuro-fuzzy control of a 4DOF upper-limb power-assist exoskeleton. In: 29th annual international conference of the IEEE EMBS, Lyon, France, August 23-26, 2007, pp 3040-3043

20. Ando T, Okamoto J, Fujie MG (2008) Intelligent corset to support rollover of cancer bone metastasis patients. In: IEEE/RSJ IEEE/RSJ international conference on intelligent robots and systems, Nice, France, September 22-26, 2008, pp 723-728

21. Fleischer C, Hommel G (2008) A human-exoskeleton interface utilizing electromyography. IEEE Trans Robot 24(4):872-882

22. Artemiadis PK, Kyrialopoulos KJ (2010) EMG-based control of a robot arm using low-dimensional embeddings. IEEE Trans Robot 26(2):393-398

23. Pau JWL, Xie SSQ, Pullan AJ (2012) Neuromuscular interfacing: establishing an EMG-driven model for the human elbow joint. IEEE Trans Biomed Eng 59(9):2586-2593

24. Cipriani C, Zaccone F, Micera S, Carrozza MC (2008) On the shared control of an EMG-controlled prosthetic hand; analysis of user-prosthesis interaction. IEEE Trans Robot 24(1):170-184

25. Deuschl G, Raethjen J, Lindermann M (2001) The pathophysiology of tremor. Muscle Nerve 24:716-735

26. Milanov I (2001) Electromyographic differentiation of tremors. Clin Neurophysiol 112(9):1626-1632

27. Journee HL (1983) Demodulation of amplitude modulated noise: a mathematical evaluation of a demodulator for pathological tremor EMGs. IEEE Trans Biomed Eng 30(5):304-308
28. Bacher M, Scholz E, Diener HC (1989) 24 hour continuous tremor quantification based on EMG recording. Neurophysiology 72(2):176-183

29. Rocon E, Belda-Lois JM, Ruiz AF, Manto M, Moreno JC, Pons JL (2007) Design and validation of a rehabilitation robotic exoskeleton for tremor assessment and suppression. IEEE Trans Neural Syst Rehabil Eng 15(3):367-378

30. Riviere CN, Ang W-T, Khosla PK (2003) Toward active tremor canceling in handheld microsurgical instruments. IEEE Trans Robot Autom 15:793-800

31. Liu F, Stern R, Huang X, Acero A (1993) Efficient cepstral normalization for robust speech recognition. In: Proceedings of the workshop on Human Language Technology, Stroudsburg, PA, USA, pp 69-74

32. Sartori M, Lloyd DG, Reggiani M, Pagello E (2009) A stiff tendon neuromusculoskeletal of the knee. In: IEEE workshop on advanced robotics and its social impacts, Tokyo, Japan, November 23-25, pp 132-138

33. Koo TKK, Mak AFT (2005) Feasibility of using EMG driven neuromusculoskeletal model for prediction of dynamic movement of the elbow. J Electromyogr Kinesiol 15:12-26

34. Cavallaro E, Rosen J, Perry JC, Burns S, Hannaford B (2005) Hill-based model as a myoprocessor for a neural controlled powered exoskeleton arm-parameters optimization. In: International conference on robotics and automation, Barcelona, Spain, April 2005, pp 4514-4519

35. Ae M, Tang HP, Yokoi T (1992) Estimation of inertia properties of the body segments in Japanese athletes. Biomechanism 11:23-33 (Japanese article)

36. Shao Q, Bassett DN, Manal K, Buchanan TS (2009) An EMG-driven model to estimate muscle forces and joint moments in stroke patients. Comput Biol Med 39(12):1083-1088

\section{Submit your manuscript to a SpringerOpen ${ }^{\circ}$ journal and benefit from:}

- Convenient online submission

- Rigorous peer review

- Immediate publication on acceptance

- Open access: articles freely available online

- High visibility within the field

- Retaining the copyright to your article

Submit your next manuscript at $\boldsymbol{\nabla}$ springeropen.com 